J. Product. \& Dev., 26(4): 1017- 1031 (2021)

\title{
IMPACT OF FEED RESTRICTED SYSTEM AND ZINC FORMS ON SOME PRODUCTIVE PERFORMANCE TRAITS, EGG QUALITY PARAMETERS AND ECONOMIC EFFICIENCY OF MANDARAH LAYING HENS.
}

\author{
Wesam A. Ibrahim*; Tawfeek I.M**; Rashwan A.A**; Abd-M.I.Rahim ** \\ and A.M.A. Bealish* \\ *Animal Production Research Institute., Agriculture Research Centre, Ministry \\ of Agriculture, Dokki, Giza, Egypt. \\ ** Animal \& Poultry Production Department, Faculty of Technology \& \\ Development, Zagazig University, Zagazig, Egypt. \\ e mail:mostawms@hotmail.com,rashwanali@hotmail.com,mostafarahim@ymail.com
}

\section{ABSTRACT:}

This study was conducted to determine the effect of feeding different level of restricted feed with different forms of zinc(Zn) on productive performance, egg quality traits and economic efficiency of Mandarah (Egyptian local developed strain) laying hens. A total number of 270 laying hens +27 cocks, 24 weeks old were randomly taken to be similar in (1228.0 $\pm \mathbf{4 4 . 0} \mathbf{g})$ in the study. Birds were randomly divided into nine experimental groups,(30 hens +3 cocks in each group) and each group was contained three replicates (10 hens +1 cock / replicate). The experimental groups involved a3x3factorial arrangement, 3 zinc forms (control, organic zinc and Nano-Zn) and 3 levels of restricted system groups (ad libitum, $90 \%$ and $80 \%$ / hen / day) were fed a balanced basal diet without supplemented zinc as a control or supplemented with $50 \mathrm{mg} \mathrm{Nano}$ - zinc /Kg diet or $50 \mathrm{mg}$ zinc from zinc methionine $/ \mathrm{Kg}$ diet, respectively),during the experimental period lasted six month.

The obtained results showed that laying hens fed ad libitum / hen $/$ day and 50mg Nano-Zn/Kg diet recorded the highest $(P<.01)$ for egg production( \%), egg weight, egg mass, feed conversion / eggs and body weight compared with the control and other groups.

Dietary Nano-Zn increased significantly total body weight change, egg production (\%); egg mass and improve feed conversion / eggs, most of egg quality.

Receiving $90 \%$ feed/ hen/day recorded the best of economic efficiency. The best value of net revenue and economic efficiency of laying feeding $90 \%$ hen / day and supplemented organic zinc.

Conclusively, feeding restricted system at level of $90 \%$ and dietary 
recommended to improve productive performance traits, egg quality parameters and economic efficiency of Mandarah laying hens.

Key words: Feed restriction, zinc forms, productive traits, egg quality, economic efficiency, laying hens

\section{INTRODUCTION}

Feed management practices aiming to improve poultry industry without increasing production cost (Mateos et al., 2012). Feed restriction is one of the possible ways to control body weight of hens during laying period and maximize the metabolic rate to some extent as well as improving feed conversion and reducing feed cost. Therefore, poultry egg producers use feed restriction programs to prevent birds from getting over weighed, to delay sexual maturity, to avoid reproductive dysfunction, and to increase the egg production (Renema and Robinson, 2004).

Recently, published results have reported that the $3 / 4$ feed restriction program employed during the rearing stage provides the best performance and reproductive traits response of broiler breeder hens reared on floor pens (Carneiro et al., 2019). Moreover, Moreira et al. (2012) observed that laying hens fed 5\%restricted feed with supply of hay ad -libitum had no significant effects on the production performance of the hens and egg quality.

In previous study, a significant effect of feed restriction on egg production in Bovan Near layer strain was documented without significant effect on egg quality trait (Fasuyi and Ojo, 2012). In contrast, other investigators reported that feed restriction has no significant effect on egg number, hen-day egg production, egg weight and egg quality (Osman et al., 2010). The researchers however, found significant effect of feed restriction on yolk index and Haugh units between Hisex Brown and Bovan White strains of layers.

Zinc has significant roles in the organism because it is a cofactor of more than 200 enzymes. One of the most significant functions of zinc is related to its antioxidant role and its participation in the antioxidant defense system (Powell, 2000). Zinc deficiency provokes oxidative damage through the effect of free radical action and alters the status antioxidant enzymes and substances (Salgueiro et al., 2000). Zn-methionine or Zn-propionate had more bioavailability than inorganic zinc sources such as $\mathrm{ZnO}$ or $\mathrm{ZnSO}_{4} \mathrm{H}_{2} \mathrm{O}$ (Rahman et al., 2002). Khajaren et al. (2006) observed improvements in egg production of layers fed organic zinc.

In general, the smaller particles, are more effective in absorption, especially if the particle size is below $100 \mathrm{~nm}$, therefore, it was hypothesized 
that nanoparticles were easier to absorb compared to their inorganic or organic counterparts (Hett, 2004). Nano trace element may enter the animal's body through direct penetration; therefore, its utilization rate will be much higher than that of the inorganic trace elements (Huang et al., 2015). Sahoo et al. (2014) and Mohammadi et al. (2015) reported that the tibia $\mathrm{Zn}$ concentration in Nano $\mathrm{Zn}$ group was higher compared to that in the $\mathrm{Zn}$-sulphate group in broiler chickens.

The use of Nano minerals, such as Nano-selenium, Nano-chromium, or nano-zinc, may improve the animal production parameters, their health status and quality of products obtained from them, the conducted researches proved that Nano element form can be used better than the inorganic form, however minerals in the chelates form is used on a large scale in the feed industry (Rajendran, 2013).

Therefore, the aim of the present study was to evaluate the effect of restricted feed and different forms of dietary $\mathrm{Zn}$ supplementation on some productive performance traits and egg quality of laying hens.

\section{MATERIALS AND METHODS}

\section{Birds, management and experimental design:}

Thepresent study was carried out at Inshas Poultry Research Station, Animal Production Research Institute, Agricultural Research Center, Egypt.

A total number of 270 Mandarah (Egyptian local developed strain) laying hens+ 27 cocks, 24 weeks old was randomly taken from the farm flock, to be similar in body weight $(1228.0 \pm 44.0 \mathrm{~g})$. Birds were randomly divided into nine treatment groups (30 hens +3 cocks in each group) and then each treatment group was divided into three replicates (10 hens +1 cock /replicate). The experimental groups involved a $3 \times 3$ factorial arrangement, 3 zinc forms (control, organic zinc and Nano-Zn) and 3 levels of restricted system groups (ad libitum, $90 \%$ and $80 \%$ / hen / day) were fed a balanced basal diet without supplemented zinc (control) or supplemented with $50 \mathrm{mg}$ Nano - zinc / Kg diet or50 $\mathrm{mg}$ zinc from zinc methionine $/ \mathrm{Kg}$ diet, respectively),during the experimental period lasted six month.

All birds were housed individually in layer's rooms and maintaining in similar managerial and conditions environment with a photoperiod length of 17 $\mathrm{h}$ daily.Feed and water were provided ad libitum throughout, the experimental period (24-48 weeks of age). Experimental diets were formulated to be is nitrogenous and iso- caloric to cover the nutrients requirements as recommended by Agriculture Ministry Decree (1996) as shown in Table 1. 
Table 1: Chemical Composition and calculated analysis of the experimental basal diet

\begin{tabular}{|l|c|}
\hline \multicolumn{1}{|c|}{ Ingredients } & \% \\
\hline Yellow corn & 63.15 \\
\hline Soybean meal (44\%) & 23.29 \\
\hline Corn gluten meal (60\%) & 3.02 \\
\hline Mono calcium phosphate & 1.39 \\
\hline Lime stone & 8.40 \\
\hline Salt (Nacl) & 0.40 \\
\hline Vitamins and minerals mixture*(Without zinc) & 0.30 \\
\hline DL-methionine & 0.05 \\
\hline Total & $\mathbf{1 0 0 . 0 0}$ \\
\hline Determined analysis** & \\
\hline Crude protein (\%) & 17.00 \\
\hline Available phosphorus (\%) & 0.72 \\
\hline Calcium (\%) & 3.41 \\
\hline Lysine (\%) & 0.868 \\
\hline Methionine (\%) & 0.377 \\
\hline Methionine+ Cystine (\%) & 0.666 \\
\hline Zinc mg./kg*** & 35 \\
\hline Metabolizable energy (Kcal ME/kg diet) ${ }^{* * * *}$ & 2748 \\
\hline Each 3 kg of Vitas & 100000 \\
\hline
\end{tabular}

Each $3 \mathrm{~kg}$ of Vitamins and Minerals mixture * contains: Vit. A 10000,000 IU; Vit.D3 2000,000 IU; Vit. E 10,000 mg; Vit.K 1000 mg; Vit.B 1000 mg; Vit.B 5000 mg; Vit.B6 $1500 \mathrm{mg}$; Vit. $\mathrm{B}_{12} 10 \mathrm{mg}$; Pantothenic acid 10,000 mg; Niacin 30,000 mg; Folic acid $1000 \mathrm{mg}$; Biotin $50 \mathrm{mg}$; Choline 250,000 mg; Manganese 60,000 mg; Copper 4,000 mg; Iron 30,000mg; Iodine $300 \mathrm{mg}$; Cobalt $100 \mathrm{mg}$; $\mathrm{CaCO} 3$ to 3,000gm.

**A According To Egyptian feed composition Tables (2001)

***According To AOAC(1998)

****According To NRC (1994)

\section{Measurements:}

Body weight (BW) of bird at 24 and 48 weeks of age and change body weight (\%) was recorded. Daily and total egg number and egg weight (g) were recorded for each hen/in eavh group, while daily and total feed intake was recorded, during the experimental periods.

Egg production rate (\%) was calculated for four weeks intervals, during the production periods as egg number/hen/period for each replicate and calculated the average of the whole experimental period.

Egg mass was calculated by multiplying egg number $\mathrm{X}$ average egg weight. Feed conversion ( $\mathrm{g}$ feed /g mass and $\mathrm{Kg}$ feed/ eggs) was calculated as 
$\mathrm{Kg}$ feed consumption produced number of eggs for four weeks intervals and the whole experimental period.

Egg quality parameters were measured at the end of the experimental period, in which 5 eggs were randomly chosen from each treatment group.

External egg quality parameters such as egg weight, egg shape index (\%) were measured. However, internal egg quality parameters such as yolk index $(\%)$, albumin index (\%), Haugh units and shell thickness were calculated and percentages of egg components (yolk, albumin and shell weight / egg weight $\mathrm{x} 100)$ were determined.

Eggs were individually weighed and broken and egg shell, yolk and albumen were weighed. Egg shell thickness, including shell membranes, was measured using a micrometer at the equator. Egg shape index and yolk index values were measured according to Sauter et al. (1951). Haugh unit scores were applied from a special chart using egg weight and albumin height, which was measured by using a triple micrometer according to Haugh (1937), Kotaiah and Mohapatra (1974) and Eisen et al. (1962).

\section{Economic efficiency $($ EEF $)$ :}

Economic efficiency (\%) of egg production was calculated from the input-output analysis, which was calculated according to the price of the experimental diets and eggs produced. These values were calculated as the net revenue per unit of total cost.

\section{Statistical analysis:}

The data were statistically analyzed using SAS (2004) from all the response variables, which were subjected to factorial analysis $(3 \times 3)$ of variance according to Snedecor and Cochran (1982). To test the effect of feeding restricted system and zinc forms of bird was as following Model:

$$
\mathrm{Y}_{\mathrm{ijk}}=\mu+\mathrm{F}_{\mathrm{i}}+\mathrm{Z}_{\mathrm{j}+}(\mathrm{FZ})_{\mathrm{ij}}+\mathrm{e}_{\mathrm{ijk}}
$$

Where: $Y_{i j k}=$ Any observation, $\mu=$ Overall mean, $F_{i}=$ Feed systems $(i=1,2$ and 3$), Z_{j}=$ Zinc forms ( $(=1,2$ and 3$),(F Z) ~ i j=$ Interaction between feed restriction and zinc forms $(\mathrm{ij}=1,2 \ldots 9), \mathrm{e}_{\mathrm{ijk}}=$ Random error.

The variations among averages of the different experimental groups were calculated according to Duncan (1955).

\section{RESULTS AND DISCUSSION}

\section{Productive performance traits:}

The effect of feed restriction or zinc forms and their interaction on productive performance for the whole experimental period are shown in Table 2 . 
Table(2): Effect of feed restriction and different supplemental zinc forms on productive perfomance traits of laying hens from 24 to 48 weeks of age.

\begin{tabular}{|c|c|c|c|c|c|c|c|c|c|c|}
\hline \multicolumn{2}{|l|}{ Items } & $\begin{array}{l}\text { BIT } \\
\text { change(g) }\end{array}$ & $\begin{array}{l}\text { Egg } \\
\text { reight(g) }\end{array}$ & $\begin{array}{l}\text { Egg } \\
\text { number }\end{array}$ & $\begin{array}{l}\text { Egg mass } \\
(\mathrm{g} d \mathrm{~d})\end{array}$ & $\begin{array}{l}\text { DFI } \\
\text { (gd) }\end{array}$ & $\begin{array}{l}\text { TFI } \\
(\mathrm{kg})\end{array}$ & 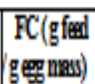 & $\begin{array}{l}\text { FC(kg feed } \\
\text { eggs) }\end{array}$ & $\begin{array}{l}\text { EPR } \\
\% \%\end{array}$ \\
\hline \multicolumn{11}{|c|}{ Feeding system } \\
\hline \multicolumn{2}{|c|}{ ad libitum } & $\begin{array}{c}335.00= \\
14.75^{2}\end{array}$ & $\begin{array}{c}49.34= \\
0.12^{2}\end{array}$ & $\begin{array}{c}104.1= \\
0.9^{4}\end{array}$ & $\begin{array}{c}30.57= \\
0.85^{2}\end{array}$ & $\begin{array}{c}118.93= \\
0.37^{2}\end{array}$ & $\begin{array}{l}19.98= \\
0.06^{2}\end{array}$ & $\begin{array}{l}3.92= \\
0.11^{1}\end{array}$ & $\begin{array}{l}5.21= \\
0.14^{b}\end{array}$ & $\begin{array}{c}61.96= \\
1.64^{4}\end{array}$ \\
\hline \multirow{2}{*}{\multicolumn{2}{|c|}{$90 \%$}} & $283.89=$ & $48.94=$ & $99.6=$ & $29.17=$ & $107.56=$ & $18.07=$ & $3.71=$ & $5.54=$ & $59.62=$ \\
\hline & & $15.89^{\circ}$ & $0.20^{25}$ & 0.72 & $0.78^{\circ}$ & $0.19^{4}$ & $0.03^{\circ}$ & $0.10^{3}$ & $0.13^{1}$ & $1.48^{\circ}$ \\
\hline \multirow{2}{*}{\multicolumn{2}{|c|}{$80 \%$}} & $230.00=$ & $48.21=$ & $88.83=$ & $24.47 \pm$ & $95.78 \pm$ & $16.09=$ & $3.93 \pm$ & $5.30=$ & $50.79=$ \\
\hline & & $19.54^{4}$ & $0.12^{\natural}$ & $0.46^{\circ}$ & $0.54^{4}$ & $0.18^{\circ}$ & $0.18^{8}$ & $0.06^{1}$ & $0.11^{2}$ & 1.07 \\
\hline \multicolumn{2}{|c|}{ Sig. test } & 44 & 44 & 44 & 44 & 444 & 444 & 44 & 4 & 44 \\
\hline \multicolumn{11}{|c|}{ Zinc forms: $1=$} \\
\hline \multicolumn{2}{|c|}{ Un-supplemented } & $\begin{array}{c}246.94= \\
17.07^{\circ}\end{array}$ & $\begin{array}{c}48.59= \\
0.25\end{array}$ & $\begin{array}{l}90.15 \pm \\
0.67^{\circ}\end{array}$ & $\begin{array}{c}25.65= \\
0.84^{4}\end{array}$ & $\begin{array}{c}107.15= \\
3.38\end{array}$ & $\begin{array}{c}18.00= \\
0.57\end{array}$ & $\begin{array}{l}4.18 z \\
0.07\end{array}$ & $\begin{array}{l}4.93= \\
0.08^{6}\end{array}$ & $\begin{array}{c}52.77= \\
1.54^{\circ}\end{array}$ \\
\hline \multicolumn{2}{|c|}{ 50mg Nano-zinc } & $\begin{array}{c}321.11= \\
18.89^{\circ}\end{array}$ & $\begin{array}{c}49.08= \\
0.19\end{array}$ & $\begin{array}{c}103.05= \\
1.01^{2}\end{array}$ & $\begin{array}{c}30.34= \\
1.05^{2}\end{array}$ & $\begin{array}{c}107.78= \\
3,37\end{array}$ & $\begin{array}{c}18.11= \\
0.57\end{array}$ & $\begin{array}{l}3.56= \\
0.04\end{array}$ & $\begin{array}{l}5.74= \\
0.07\end{array}$ & $\begin{array}{c}61.81 \pm \\
104^{4}\end{array}$ \\
\hline \multicolumn{2}{|c|}{50 mg organic dinc } & $\begin{array}{l}280.83= \\
23.42^{21}\end{array}$ & $\begin{array}{c}48.81= \\
0.19\end{array}$ & $\begin{array}{l}98.79 \pm \\
0.77^{4}\end{array}$ & $\begin{array}{c}28.22= \\
0.00^{\circ}\end{array}$ & $\begin{array}{c}107.33 \pm \\
3.30\end{array}$ & $\begin{array}{c}18.03 \pm \\
0.55\end{array}$ & $\begin{array}{l}3.81= \\
0.05^{\circ}\end{array}$ & $\begin{array}{l}5.39= \\
0.06\end{array}$ & $\begin{array}{c}57.80= \\
1.86^{\circ}\end{array}$ \\
\hline \multicolumn{2}{|c|}{ Sig. test } & tt & NS & 44 & th & NS & NS & 44 & th & t* \\
\hline \multicolumn{11}{|c|}{ Intrection effect } \\
\hline \multirow{3}{*}{ adfition } & 0 & $\begin{array}{l}294.17= \\
2043^{* 6}\end{array}$ & $\begin{array}{l}49.14= \\
0.35{ }^{25}\end{array}$ & $\begin{array}{l}95.01 \pm \\
1.27\end{array}$ & $\begin{array}{c}27.80= \\
1.25^{\circ}\end{array}$ & $\begin{array}{c}118.78= \\
0.62^{2}\end{array}$ & $\begin{array}{c}19.95 \pm \\
0.10^{1}\end{array}$ & $\begin{array}{l}4.29= \\
0.19^{4}\end{array}$ & $\begin{array}{l}4.76 \pm \\
0.19^{4}\end{array}$ & $\begin{array}{c}56.55= \\
2.27^{\circ}\end{array}$ \\
\hline & Nano-Zn & $\begin{array}{c}376.67= \\
18.33^{12}\end{array}$ & $\begin{array}{l}49.55 \pm \\
0.12^{2}\end{array}$ & $\begin{array}{c}112.02= \\
0.49^{4}\end{array}$ & $\begin{array}{c}33.03 \pm \\
0.35^{2}\end{array}$ & $\begin{array}{c}119.44= \\
0.29^{4}\end{array}$ & $\begin{array}{c}20.07= \\
0.05^{2}\end{array}$ & $\begin{array}{l}3.93 \pm \\
0.08^{a}\end{array}$ & $\begin{array}{l}5.58 \pm \\
0.09^{\circ}\end{array}$ & $\begin{array}{c}66.69= \\
0.88^{2}\end{array}$ \\
\hline & $0 \mathrm{r}-\mathrm{Zn}$ & $\begin{array}{l}334.17= \\
12.44^{21}\end{array}$ & $\begin{array}{l}49.32 \pm \\
0.05{ }^{512}\end{array}$ & $\begin{array}{c}105.27= \\
0.28^{8}\end{array}$ & $\begin{array}{c}30.89= \\
0.211^{\circ}\end{array}$ & $\begin{array}{c}118.56= \\
0.99^{4}\end{array}$ & $\begin{array}{c}19.92= \\
0.17^{2}\end{array}$ & $\begin{array}{l}4.18= \\
0.077^{1}\end{array}$ & $\begin{array}{l}5.29= \\
0.06^{\circ}\end{array}$ & $\begin{array}{c}62.66= \\
0.51^{1}\end{array}$ \\
\hline \multirow{3}{*}{$90 \%$} & 0 & $\begin{array}{l}250.00= \\
7.64^{464}\end{array}$ & $\begin{array}{c}48.63 \pm \\
0.48\end{array}$ & $\begin{array}{l}91.29= \\
0.15^{6}\end{array}$ & $\begin{array}{c}26.43^{2}= \\
0.14^{: 4}\end{array}$ & $\begin{array}{c}107.22= \\
0.40^{\circ}\end{array}$ & $\begin{array}{c}18.01= \\
0.07^{\circ}\end{array}$ & $\begin{array}{l}3.56 \pm \\
0.04^{4}\end{array}$ & $\begin{array}{l}5.07= \\
0.01^{6}\end{array}$ & $\begin{array}{c}54.35 \pm \\
0.27\end{array}$ \\
\hline & Nano-Zn & $\begin{array}{l}308.33 \pm \\
29.49^{15}\end{array}$ & $\begin{array}{l}49.32 \pm \\
0.10^{13}\end{array}$ & $\begin{array}{c}104.88= \\
0.83^{\circ}\end{array}$ & $\begin{array}{c}31.73 \pm \\
0.32^{\circ}\end{array}$ & $\begin{array}{c}107.78= \\
0.40^{\circ}\end{array}$ & $\begin{array}{c}18.11= \\
0.07^{\circ}\end{array}$ & $\begin{array}{l}3.81= \\
0.05^{\circ}\end{array}$ & $\begin{array}{l}5.98= \\
0.08^{2}\end{array}$ & $\begin{array}{c}64.40= \\
0.79^{\circ}\end{array}$ \\
\hline & $0 \mathrm{r}-\mathrm{Zn}$ & $\begin{array}{l}293.33 \pm \\
34.44^{204}\end{array}$ & $\begin{array}{l}48.87= \\
0.31^{126}\end{array}$ & $\begin{array}{c}101.01= \\
0.03^{*}\end{array}$ & $\begin{array}{c}29.35= \\
0.22^{\circ}\end{array}$ & $\begin{array}{c}107.67= \\
0.19^{\circ}\end{array}$ & $\begin{array}{c}18.09= \\
0.03^{\circ}\end{array}$ & $\begin{array}{l}4.29= \\
0.19^{1}\end{array}$ & $\begin{array}{l}5.58 \pm \\
0.02^{4}\end{array}$ & $\begin{array}{c}60.12= \\
0.06^{\circ}\end{array}$ \\
\hline \multirow{3}{*}{$80 \%$} & 0 & $\begin{array}{c}196.67= \\
25.22^{6}\end{array}$ & $\begin{array}{c}48.01= \\
0.3 l_{6}\end{array}$ & $\begin{array}{l}84.12 \pm \\
0.589\end{array}$ & $\begin{array}{c}22.74= \\
0.28^{\circ}\end{array}$ & $\begin{array}{c}9.45 \pm \\
0.29^{\circ}\end{array}$ & $\begin{array}{c}16.03= \\
0.05:\end{array}$ & $\begin{array}{l}3.62= \\
0.05^{\text {t4 }}\end{array}$ & $\begin{array}{l}4.97= \\
0.09\end{array}$ & $\begin{array}{c}47.42= \\
0.80^{4}\end{array}$ \\
\hline & Nano-Zn & $\begin{array}{l}\text { nas } \\
\text { atsod }\end{array}$ & $\begin{array}{l}48.37 \pm \\
0.09^{2 * 6}\end{array}$ & $\begin{array}{l}92.28= \\
0.25^{6}\end{array}$ & $\begin{array}{l}26.27 \pm \\
0.23^{64}\end{array}$ & $\begin{array}{c}96.11= \\
0.29^{\circ}\end{array}$ & $\begin{array}{l}16.15= \\
0.055^{\circ}\end{array}$ & $\begin{array}{l}3.84= \\
0.04^{*}\end{array}$ & $\begin{array}{l}5.65= \\
0.04^{\circ}\end{array}$ & $\begin{array}{c}54.33= \\
0.56^{\circ}\end{array}$ \\
\hline & Or-Zn & $\begin{array}{l}215.00= \\
39.69^{\mathrm{\alpha}}\end{array}$ & $\begin{array}{l}48.13= \\
0.25^{*}\end{array}$ & $\begin{array}{l}90.06= \\
0.40^{\circ}\end{array}$ & $\begin{array}{l}24.41= \\
0.43^{6}\end{array}$ & $\begin{array}{c}95.78= \\
0.29^{\circ}\end{array}$ & $\begin{array}{c}16.09= \\
0.05:\end{array}$ & $\begin{array}{l}4.06= \\
0.04^{8}\end{array}$ & $\begin{array}{l}5.29= \\
0.12^{\circ}\end{array}$ & $\begin{array}{c}50.63= \\
0.9\end{array}$ \\
\hline & & t\$ & t\$ & 44 & th & 444 & 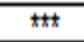 & 44 & 4 & t\$ \\
\hline
\end{tabular}

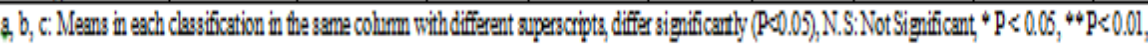

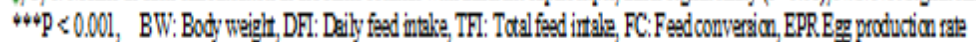


Feeding ad libitum of the diet significantly $(\mathrm{P} \leq 0.01)$ improved egg production percentage, egg mass and body weight change of layer compared with $90 \%$ or $80 \%$ hens. The best feed conversion ratio for laying was recorded with birds fed $90 \%$ hens as compared with those fed $80 \%$ or ad libitum / hens.

Nano-Zn supplementation in layer diets significantly $(\mathrm{P} \leq 0.01)$ increased egg production percentage, egg mass and body weight change and improved feed conversion ratio compared with organic $\mathrm{Zn}$ and un- $\mathrm{Zn}$ supplementation.

Table (2) show that egg production percentage, egg mass, body weight change and feed conversion were significantly $(\mathrm{P} \leq 0.01)$ influenced by interaction between feed restriction and zinc forms. On the other hand, the egg weight was not significantly affected by the interaction between feed restriction and zinc forms.

These results agreed with Souza et al. (2005) who found that the poultry production in the free-range system to be feasible should be directed to the use of alternative feeding and pastures, in the free-range system. The same author added that feeding of birds with free commercial diet may cause losses, even selling the eggs with price higher than the recommended for eggs produced industrially. These findings are in line with Abedini et al., (2018) who observed the positive effect of dietary supplementation with Nano $\mathrm{Zn}$ on egg production in laying hens. Higher bioavailability of $\mathrm{Zn}$ in the form $\mathrm{ZnO}-\mathrm{NPs}$ could explain the improved egg production. El-Katcha et al. (2018) reported that Zn-NPs significantly $(\mathrm{P}<0.05)$ increased the average egg production\% throughout the whole experimental periods compared to the control. Guo et al. (2002) reported no effect on egg production in laying hens fed diets supplemented with $(60 \mathrm{mg} / \mathrm{kg})$ inorganic zinc (zinc sulfate). The significant increase in egg production of the Nano zinc $(60 \mathrm{mg} / \mathrm{kg})$ group might be due to the important role of $\mathrm{Zn}$ in the synthesis and secretion of LH and FSH hormones (Bedwal and Bahugana, 1994).

Dietary zinc may influence egg production by interacting with the endocrine system since the hen is changing the production and secretion of reproductive hormones during sexual maturation (Renema et al., 1999). Olgun and Yildiz (2017) reported a contrast result that supplementation $75 \mathrm{mg} / \mathrm{kg}$ Nano-Zn did not affect egg production rate of laying hens. On the other hand, $75 \mathrm{mg} / \mathrm{kg}$ organic zinc supplementation increased egg production \% compared with laying hens fed on the same level of supplemental zinc from nano source. These results of the present study are supported by Bahakaim et al., (2014) who indicated that the EM increased with the addition of organic $\mathrm{Zn}$ (Zn-methionine) compared to Zn-sulfate in laying hens. Also, Abd El-Hack et al. (2017) who reported that replacement of inorganic zinc by zinc-methionine significantly 
increased egg mass of laying hens. Moreover, organic zinc increased average EM as compared with birds fed supplemental zinc from Nano source.

\section{Egg quality parameters:}

The effect of Feed restriction supplemental or zinc forms and their interaction on external and internal egg quality is shown in Table (3) All parameters were significantly $(\mathrm{P}<.01)$ affected by $\mathrm{Zn}$ supplementation egg quality except, of Egg shape index shell weight and yolk index which was insignificantly affected by feed restriction system.

All egg quality parameters were significantly $(\mathrm{P} \leq 0.05)$ affected by the interaction among feed restriction system and $\mathrm{Zn}$ forms, except of egg shape index. Zinc plays an important role in the isthmus, where egg shell membranes are produced. Dietary zinc improved egg shell quality because $\mathrm{Zn}$ is a component of the carbonic anhydrase enzyme, which is necessary for the formation of egg shell (Carneiro et al., 2019 and Innocenti et al., 2004). Konkol and Wojnarowski (2018) showed that thickness of the egg shell was significantly $(\mathrm{P}<0.05)$ higher in groups birds receiving Nano-zinc and zinc methionine $60 \mathrm{mg} / \mathrm{kg}$ diet than the control group. Abd El-Hack et al.,(2017) observed that the highest values of Haugh unit scores and yolk to albumin ratio were recorded by the control group, which fed diet free of $\mathrm{ZnO}$ or $\mathrm{Zn}-\mathrm{Met}$. Supplementation with Nano or organic- Zn sources showed were improvement in shell thickness. These results were in agreement with the findings of Yang et al. (2012) who reported that $\mathrm{Zn}$ supplementation $(65 \mathrm{mg} / \mathrm{kg})$ organic increased egg shell thickness.

Another reason might be attributed as Zinc plays a role in the magnum during the deposition of albumen and in the isthmus where eggs hell membranes are produced. The increase in weight of albumen in the treated group as compared to the control group were also reported by Tabatabaie et al., (2007) showed that supplementation the diet with organic zinc at a different level. Among different $\mathrm{Zn}$ sources used in the study, nano zinc particles are the better source in which bioavailability is highest, due to their extremely small size and unique physical properties (Sahoo et al., 2014). The increase of shell thickness might be attributed to the importance of zinc function in the formation of the egg. Zinc supplementation also has been reported to improve egg shell quality because it is a component of the carbonic anhydrase enzyme, which supplies with the carbonate ions during eggshell formation (Innocenti et al., 2004). Supplementation of nano zinc gave the preferable value in the Haugh unit. The reason might be attributed to an increase in albumen weight. 
Table (3): Egg quality parametwers $(\bar{X} \pm S E)$ of Mandarah hens as affected by different forms of zinc and feed restriction and their interactions at 48 weeks of age.

\begin{tabular}{|c|c|c|c|c|c|c|c|c|c|c|}
\hline \multirow{2}{*}{\multicolumn{2}{|c|}{ Items }} & \multicolumn{2}{|c|}{ External egg quality } & \multicolumn{4}{|c|}{$\begin{array}{c}\text { Internal egg quality } \\
\text { indices }\end{array}$} & \multicolumn{3}{|c|}{$\begin{array}{c}\text { Component of egg weight } \\
(\%)\end{array}$} \\
\hline & & $\begin{array}{c}\text { Egg } \\
\text { Weight } \\
\text { (g) }\end{array}$ & $\begin{array}{c}\text { Egg shape } \\
\text { index } \\
(\%)\end{array}$ & $\begin{array}{c}\text { Sell } \\
\text { thickens } \\
(\mathbf{m m})\end{array}$ & $\begin{array}{c}\text { Yolk } \\
\text { index } \\
(\%)\end{array}$ & $\begin{array}{l}\text { Albumen } \\
\text { index } \\
(\%)\end{array}$ & $\begin{array}{c}\text { Haug } \\
\text { h unit } \\
\text { (score) }\end{array}$ & $\begin{array}{c}\text { Yolk } \\
\text { weight } \\
(\%)\end{array}$ & $\begin{array}{c}\text { Albumen } \\
\text { weight } \\
(\%)\end{array}$ & $\begin{array}{c}\text { Shell } \\
\text { weight } \\
(\%)\end{array}$ \\
\hline \multicolumn{11}{|c|}{ Feeding restricted system } \\
\hline \multicolumn{2}{|c|}{$\begin{array}{l}\text { ad } \\
\text { libitum }\end{array}$} & $\begin{array}{c}51.87 \pm 0 \\
17\end{array}$ & $\begin{array}{c}78.18 \pm \\
0.46\end{array}$ & $\begin{array}{l}0.42 \pm \\
0.003^{\mathrm{a}}\end{array}$ & $\begin{array}{l}46.03 \pm \\
0.22^{\mathrm{a}}\end{array}$ & $9.82 \pm$ & $\begin{array}{l}81.22 \pm \\
0.59^{\mathrm{a}}\end{array}$ & $\begin{array}{l}33.67 \pm \\
0.06^{\mathrm{c}}\end{array}$ & $\mathrm{a}^{\mathrm{o}}$ & $\begin{array}{l}10.16 \pm \\
0.05^{\mathrm{a}}\end{array}$ \\
\hline \multicolumn{2}{|c|}{$90 \%$} & 21 & & $\begin{array}{l}0.41 \pm \\
0.004^{b}\end{array}$ & $0.33^{\mathrm{a}}$ & & $0.56^{\mathrm{b}}$ & $\begin{array}{c}33.94 \pm \\
0.06^{\mathrm{b}}\end{array}$ & & $\begin{array}{r}10.03 \pm \\
0.05^{\mathrm{a}}\end{array}$ \\
\hline \multicolumn{2}{|c|}{$80 \%$} & $\begin{array}{c}51.46 \pm \\
0.279\end{array}$ & $\begin{array}{c}76.66 \pm \\
0.63\end{array}$ & $\begin{array}{l}0.38 \pm \\
0.002^{\mathrm{c}}\end{array}$ & $\begin{array}{l}44.56 \pm \\
0.26^{\mathrm{b}}\end{array}$ & $\begin{array}{l}8.10 \pm \\
0.85^{\mathrm{b}}\end{array}$ & $\begin{array}{c}77.56 \pm \\
0.47^{c}\end{array}$ & $\begin{array}{l}34.13 \pm \\
0.07^{\mathrm{a}}\end{array}$ & $\begin{array}{l}56.00 \pm \\
0.05^{\mathrm{b}}\end{array}$ & $\begin{array}{l}9.87 \pm \\
0.05^{\mathrm{b}}\end{array}$ \\
\hline Sig. & & & & $* *$ & $* *$ & $* *$ & $* *$ & $* *$ & 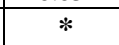 & $*$ \\
\hline \multicolumn{11}{|c|}{ Zinc forms } \\
\hline \multicolumn{2}{|c|}{$\begin{array}{l}\text { Un-suppl. } \\
\text { (1) }\end{array}$} & $\begin{array}{c}51.77 \pm \\
0.20\end{array}$ & $\begin{array}{c}77.81 \pm \\
0.46\end{array}$ & $\begin{array}{l}0.39 \pm \\
0.004^{b}\end{array}$ & $\begin{array}{c}45.11 \pm \\
0.31\end{array}$ & $\begin{array}{l}8.90 \pm \\
0.225^{\mathrm{b}}\end{array}$ & $\begin{array}{c}77.31 \pm \\
0.44^{\mathrm{c}}\end{array}$ & $\begin{array}{c}34.08 \pm \\
0.06^{\mathrm{a}}\end{array}$ & $\begin{array}{c}55.96 \pm \\
0.04^{\mathrm{b}}\end{array}$ & $\begin{array}{c}9.96 \pm \\
0.06\end{array}$ \\
\hline \multicolumn{2}{|c|}{$\begin{array}{l}50 \text { mg Nano } \\
\text { zinc(2) } \\
\end{array}$} & $\begin{array}{c}51.73 \pm \\
0.30 \\
\end{array}$ & $\begin{array}{c}77.10 \pm \\
0.87 \\
\end{array}$ & $\begin{array}{l}0.41 \pm \\
0.005^{\mathrm{a}} \\
\end{array}$ & $\begin{array}{c}45.83 \pm \\
0.28 \\
\end{array}$ & $\begin{array}{c}9.44 \pm 0.2 \\
21^{\mathrm{a}} \\
\end{array}$ & $\begin{array}{c}81.46 \pm \\
0.58^{\mathrm{a}} \\
\end{array}$ & $\begin{array}{c}33.76 \pm \\
0.07^{\mathrm{b}} \\
\end{array}$ & $\begin{array}{c}56.16 \pm \\
0.05^{\mathrm{a}} \\
\end{array}$ & $\begin{array}{c}10.09 \pm \\
0.06\end{array}$ \\
\hline \multicolumn{2}{|c|}{$\begin{array}{l}50 \mathrm{mg} \text { org. } \\
\text { zinc(3) }\end{array}$} & $\begin{array}{c}51.58 \pm \\
0.23\end{array}$ & $\begin{array}{r}77.2 \\
0.5\end{array}$ & $\begin{array}{l}0.40 \pm \\
0.005^{\mathrm{a}}\end{array}$ & $\begin{array}{c}45.25 \pm \\
0.33\end{array}$ & $\begin{array}{c}9.22 \pm 0.2 \\
18^{\mathrm{a}}\end{array}$ & $\begin{array}{c}80.26 \pm \\
0.46^{\mathrm{b}}\end{array}$ & $\begin{array}{c}33.90 \pm \\
0.08^{\mathrm{b}}\end{array}$ & $\begin{array}{c}56.06 \pm \\
0.05^{\mathrm{ab}}\end{array}$ & $\begin{array}{c}10.02 \pm \\
0.05\end{array}$ \\
\hline Sig. & & $\mathbf{N}$ & NS & $* *$ & NS & 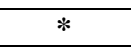 & $* *$ & $* *$ & $*$ & NS \\
\hline \multicolumn{11}{|c|}{ Interaction effect } \\
\hline \multirow{3}{*}{$\begin{array}{l}\text { ad } \\
\text { lib. }\end{array}$} & (1) & $\begin{array}{c}51.94 \pm \\
0.38\end{array}$ & $\begin{array}{c}77.89 \pm \\
0.55\end{array}$ & $\begin{array}{c}0.40 \pm \\
0.004^{\mathrm{bc}}\end{array}$ & $\begin{array}{l}45.74 \pm \\
0.56^{\mathrm{abc}}\end{array}$ & $\begin{array}{c}9.52 \pm \\
0.099^{\mathrm{bc}}\end{array}$ & $\begin{array}{c}78.36 \pm \\
0.48^{\mathrm{c}}\end{array}$ & $\begin{array}{l}33.91 \pm \\
0.04^{\mathrm{bc}}\end{array}$ & $\begin{array}{c}55.98 \pm \\
0.09^{\mathrm{c}}\end{array}$ & $\begin{array}{l}10.10 \pm \\
0.08^{\mathrm{ab}}\end{array}$ \\
\hline & (2) & $\begin{array}{c}51.82 \pm \\
0.52\end{array}$ & $\begin{array}{c}78.73 \pm \\
0.78\end{array}$ & $\begin{array}{c}0.43 \pm \\
0.004^{\mathrm{a}}\end{array}$ & $\begin{array}{r}46.41 \pm \\
0.36^{\mathrm{a}}\end{array}$ & $\begin{array}{l}10.11 \pm \\
0.039^{\mathrm{a}}\end{array}$ & $\begin{array}{c}83.35 \pm \\
0.36^{\mathrm{a}}\end{array}$ & $\begin{array}{c}33.50 \pm \\
0.09^{\mathrm{e}}\end{array}$ & $\begin{array}{c}56.26 \pm \\
0.06^{\mathrm{a}}\end{array}$ & $\begin{array}{r}10.23 \pm \\
0.10^{\mathrm{a}}\end{array}$ \\
\hline & (3) & $\begin{array}{c}51.85 \pm \\
0.27 \\
\end{array}$ & $\begin{array}{c}77.91 \pm \\
1.11 \\
\end{array}$ & $\begin{array}{l}0.42 \pm \\
0.003^{\mathrm{a}} \\
\end{array}$ & $\begin{array}{c}45.94 \pm \\
0.06^{\mathrm{ab}} \\
\end{array}$ & $\begin{array}{c}9.84 \pm \\
0.156^{\mathrm{abc}} \\
\end{array}$ & $\begin{array}{c}81.95 \pm \\
0.25^{\mathrm{a}} \\
\end{array}$ & $\begin{array}{c}33.59 \pm \\
0.066^{\mathrm{de}}\end{array}$ & $\begin{array}{c}56.24 \pm \\
0.07^{\mathrm{ab}}\end{array}$ & $\begin{array}{c}10.16 \pm \\
0.06^{\mathrm{a}} \\
\end{array}$ \\
\hline \multirow{3}{*}{$\begin{array}{l}90 \\
\%\end{array}$} & (1) & $\begin{array}{r}51.5 \\
0.2\end{array}$ & $\begin{array}{c}78.60 \pm \\
0.63\end{array}$ & $\begin{array}{c}0.39 \pm \\
0.005^{\mathrm{de}}\end{array}$ & $\begin{array}{l}45.16 \pm \\
0.47^{\mathrm{abc}}\end{array}$ & $\begin{array}{l}9.41 \pm \\
0.193^{\mathrm{c}}\end{array}$ & $\begin{array}{c}78.05 \pm \\
0.42^{\mathrm{c}}\end{array}$ & $\begin{array}{l}34.09 \pm \\
0.08^{\mathrm{abc}}\end{array}$ & $\begin{array}{c}55.95 \pm \\
0.01^{\mathrm{c}}\end{array}$ & $\begin{array}{c}9.97 \pm \\
0.11\end{array}$ \\
\hline & (2) & $\begin{array}{r}51.7 \\
0.4\end{array}$ & $\begin{array}{r}76.0 \\
1.9\end{array}$ & $\begin{array}{l}0.42 \pm \\
0.006^{\mathrm{a}}\end{array}$ & $\begin{array}{c}46.10 \pm \\
0.48^{\mathrm{a}}\end{array}$ & $\begin{array}{c}9.89 \pm \\
0.142^{\text {ab }}\end{array}$ & $\begin{array}{c}82.28 \pm \\
0.54^{\mathrm{a}}\end{array}$ & $\begin{array}{c}33.81 \pm \\
0.09^{\text {cd }}\end{array}$ & $\begin{array}{l}56.12 \pm \\
0.12^{\text {abc }}\end{array}$ & $\begin{array}{l}10.09 \pm \\
0.05^{\mathrm{abc}}\end{array}$ \\
\hline & (3) & $\begin{array}{c}51.63 \pm \\
0.49\end{array}$ & $\begin{array}{c}77.29 \pm \\
1.33\end{array}$ & $\begin{array}{c}0.41 \pm \\
0.005^{\mathrm{ab}}\end{array}$ & $\begin{array}{c}45.52 \pm \\
0.76^{\mathrm{abc}}\end{array}$ & $\begin{array}{c}9.60 \pm \\
0.260^{b c}\end{array}$ & $\begin{array}{c}80.45 \pm \\
0.74^{\mathrm{b}}\end{array}$ & $\begin{array}{c}33.92 \pm \\
0.13^{\mathrm{bc}}\end{array}$ & $\begin{array}{c}56.00 \pm \\
0.07^{\mathrm{bc}}\end{array}$ & $\begin{array}{l}10.04 \pm \\
0.11^{\mathrm{abc}}\end{array}$ \\
\hline \multirow{3}{*}{$\begin{array}{l}80 \\
\%\end{array}$} & (1) & $\begin{array}{c}51.46 \pm \\
0.50\end{array}$ & $\begin{array}{c}76.94 \pm \\
0.98\end{array}$ & $\begin{array}{l}0.37 \pm \\
0.005^{\mathrm{e}}\end{array}$ & $\begin{array}{c}44.43 \pm \\
0.41^{\mathrm{bc}}\end{array}$ & $\begin{array}{l}7.78 \pm \\
0.131^{\mathrm{e}}\end{array}$ & $\begin{array}{c}75.52 \pm \\
1.09^{\mathrm{d}}\end{array}$ & $\begin{array}{c}34.24 \pm \\
0.17^{\mathrm{a}}\end{array}$ & $\begin{array}{c}55.95 \pm \\
0.12^{\mathrm{c}}\end{array}$ & $\begin{array}{c}9.81 \pm \\
0.07^{c}\end{array}$ \\
\hline & (2) & $\begin{array}{c}51.65 \pm \\
0.66\end{array}$ & $\begin{array}{c}76.56 \pm \\
1.63\end{array}$ & $\begin{array}{c}0.39 \pm \\
0.003^{\text {de }}\end{array}$ & $\begin{array}{l}44.97 \pm \\
0.43^{\mathrm{abc}}\end{array}$ & $\begin{array}{c}8.31 \pm \\
0.080^{d}\end{array}$ & $\begin{array}{c}78.75 \pm \\
0.48^{\mathrm{c}}\end{array}$ & $\begin{array}{c}33.97 \pm \\
0.11^{\mathrm{abc}}\end{array}$ & $\begin{array}{l}56.10 \pm \\
0.07^{\text {abc }}\end{array}$ & $\begin{array}{l}9.95 \pm \\
0.11^{\mathrm{abc}}\end{array}$ \\
\hline & (3) & $\begin{array}{c}51.25 \pm \\
0.42\end{array}$ & $\begin{array}{c}76.49 \pm \\
0.63\end{array}$ & $\begin{array}{c}0.38 \pm \\
0.004^{\text {de }}\end{array}$ & $\begin{array}{c}44.29 \pm \\
0.44^{\mathrm{c}}\end{array}$ & $\begin{array}{l}8.21 \pm \\
0.136^{\mathrm{d}}\end{array}$ & $\begin{array}{c}78.39 \pm \\
0.24^{\mathrm{c}}\end{array}$ & $\begin{array}{c}34.18 \pm \\
0.06^{\mathrm{ab}}\end{array}$ & $\begin{array}{c}55.95 \pm \\
0.06^{\mathrm{c}}\end{array}$ & $\begin{array}{l}9.85 \pm \\
0.06^{\mathrm{bc}}\end{array}$ \\
\hline \multicolumn{2}{|c|}{ Sig.test } & NS & NS & $* *$ & $*$ & NS & $* *$ & $* *$ & $* *$ & $*$ \\
\hline
\end{tabular}

a,b,c: Means in the same column with different superscripts, differ significantly $(\mathrm{P}<0.05)$ N.S: Not Significant, * $\mathrm{P}<0.05, * * \mathrm{P}<0.01$. ad lib :ad libitum 


\section{Economic efficiency:}

Results of economic efficiency (\%) are summarized in Table (4). Regardless feeding of (90\% from ad libitum / hen / day) increased the net revenue over than feeding $80 \%$ from ad libitum / hen / day. Whereas, feeding of organic zinc increased the net revenue per hen over than the Nano zinc and un supplemented group.

Concerning the effect of interaction between feed restriction and zinc forms showed that the best value of net revenue and economic efficiency of laying feeding $90 \%$ hen / day supplemented organic zinc. Olawumi (2014) found that $90 \%$ ad libitum was better and feed efficient than ad libitum and $80 \%$ ad libitum recorded higher net returns and economic efficiency. These results of the present study suggested that the quantitative feed restriction (90\%/hen /day) contain $50 \mathrm{mg}$. Organic zinc is employed to control growth by feeding a predetermined amount of balanced diet in order to achieve a good production during laying period as well enhanced the economic efficiency.

Conclusively, feeding restricted system at level of $90 \%$ and dietary Organic-Zn supplementation at level of $50 \mathrm{mg} / \mathrm{kg}$ can be recommended to improve productive performance traits, egg quality parameters and economic efficiency of Mandarah laying hens.

\section{REFERENCES}

Abd El-Hack, M. E., Alagawany, M., Salah, A. S., Abdel-Latif, M. A., Farghly, M. F. A. (2017).Effects of Dietary Supplementation of Zinc Oxide and Zinc Methionine on Layer Performance, Egg Quality, and Blood Serum Indices. Biol. Trace Elem. Res., 184(2): 456-462.

Abedini, M., Shariatmadari, F., Torshizi, M. A. K., Ahmadi, H. (2018).Effects of Zinc Oxide Nanoparticles on Performance, Egg Quality, Tissue Zinc Content, Bone Parameters, and Antioxidative Status in Laying Hens. Biol. Trace Elem. Res., 184(1): 259-267.

Agriculture Ministry Decree, (1996).The standard properties for ingredients, feed additives and feed manufactured for animal and poultry. El-Wakaee El- Masria, No. 192 (1997) pp. 95 Amirria Press, Cairo, Egypt.

A.O.A.C. (1998). Association of Official Analytical Chemists, Official Methods of Analysis. $15^{\text {th }}$ Edition, Published by the AOAC, Washington, D. C., USA. 


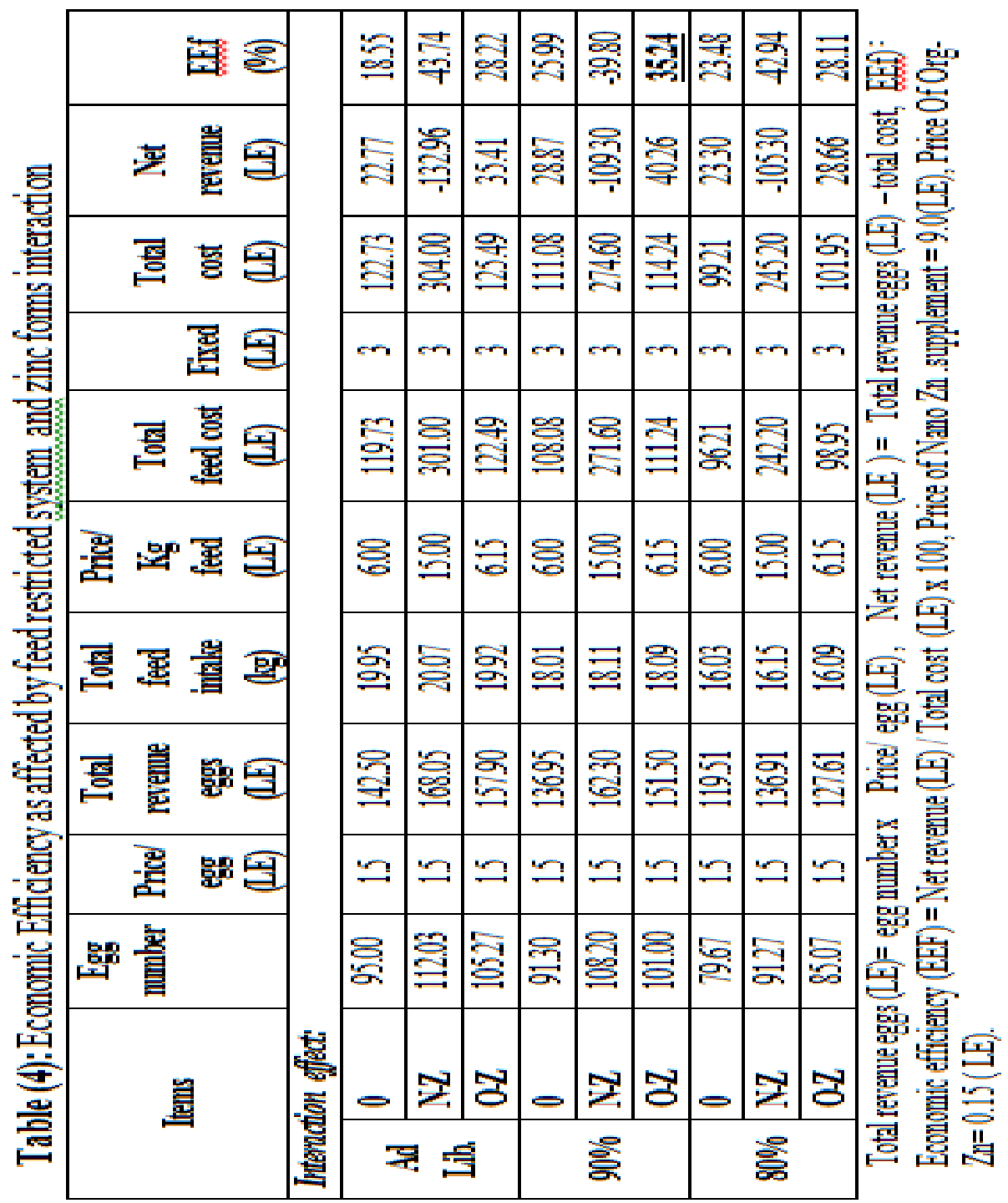


Bahakaim, A. S. A., Abdel, H. A., Magied, S. M. H., Osman, Omar, A. S. Abdelmalak, N. Y. and Nehad, A.R. (2014).Effect of using different levels and sources of zinc in layer's diets on egg zinc enrichment. Egypt. Poultry Sci., 34(i): 39-56.

Bedwal, R. S. and Bahugana, A.(1994). Zinc, copper and selenium in reproduction. Experiential., 50: 626-640.

Carneiro, P. R. O.; Lunedo, R.,; Fernandez-Alarcon, M. F.; Baldissera, G.; Freitas, G. G. and Macari, M. (2019).Effect of different feed restriction programs on the performance and reproductive traits of broiler breeders. Poultry Science, 98:4705-4715.

Duncan, D.B. (1955).Multiple ranges and multiple F-tests.Biometrics, 11:1-42.

Eisen, E.J.; B.B. Bohren and M. Mckean (1962). The Hough Unit as a measure of egg albumin quality. Poultry Sci., 41: 1461.

El-Katcha M; Soltan MA; El-badry M. (2018).Impact of Dietary Replacement of Inorganic Zinc by Organic or Nano Sources on Productive Performance, Immune Response and Some Blood Biochemical Constituents of Laying Hens. Alex. J. Vet. Sci., 59:45-59. https://doi.org/10. 5455/ajvs.266925

Fasuyi, A. O. and O. G. Ojo. (2012). Quantitative feed restriction threshold in Bovan Nera laying pullets in the warm wet tropics". J. Animal Sci. Advances, 2(1), pp. 158-165.

Guo, Y. M.; Yang, R.; Yuan, J.; Ward, T. L. and Fakler, T. M. (2002). Effect of Availar-Zn and Znso4 on laying hen performance and egg quality. Poultry Sci., 81 (Suppl. 1): 40. (Abstr.).

Haugh, R.R., (1937) . The Haugh unit for measuring egg quality. United States, Egg Poultry Magazine, 43: 552- 555.

Hett A. (2004). Nanotechnology: Small matter. Many unknowns. Swiss Reinsurance Company, Zurich.

Huang S., Wang L., Liu L., Hou Y., Li L. (2015). Nanotechnology in agriculture, livestock, and aquaculture in China. A Review. Agron. Sustain. Dev., 35: 369-400.

Innocenti, A., Zimmerman, S., Ferry, J. G., Scozzafava, A. and Supuran, C. T. (2004). Carbonic anhydrase inhibitors.Inhibition of the zinc and cobalt gamma class enzyme from the archaenon methanosarcina thermophila with anions. Bioor. Med. Chem. Lett., 14: 3327-3331. 
Khajaren, J., S. Khajaren, C. J. Rapp, T. A. Ward, A. Johnson and T.M. Falker (2006).Effects of zinc and manganese amino acid complexes (Availa-Z/M) on layer production and egg quality. http://us. znpro. com/ research/ZPA/ZPA0048.htm.

Konkol, D. and K. Wojnarowski (2018). The Use of Nano sminerals in Animal Nutrition as a Way to Improve the Composition and Quality of Animal Products. Journal of Chemistry, Article ID 5927058, 7 pages.

Kotaiah, T. and S.C. Mohapatra (1974).Measurement of albumin quality. India Poult. Ganzette., 59: 121.

Mateos, G. G., Jimenez-Moreno,E., Serrano,M. P.and_azaro,R. L.2012. Poultry response to high levels of dietary fiber sources varying in physical and chemical characteristics. J. Appl. Poult. Res. 21:156-174.

Mohammadi V.; Ghazanfari S.; Mohammadi-Sangcheshmeh S.; Nazaran M.H. (2015). Comparative effects of zinc-nano complexes, zincsulphate and zinc methionine on performance in broiler chickens. Brit. Poultry Sci., 56: 486-493.

Moreira, R.F.,; Freitas, E.R.; Sueupira, F.S.; Freitas Diogenes, A.L.S.; Abe, M.S and Araujo, F.W.S. (2012).Effect of feed restriction with voluntary hay intake on the performance and quality of laying hen eggs. Acta Scientiarum. Animal Science, 34 (2): 149-154.

Olgun, O. and A. O. Yildiz (2017). Effects of dietary supplementation of inorganic, organic or nano zinc forms on performance, eggshell quality, and bone characteristics in laying hens. Ann. Anim. Sci., 17(2): 463-476.

Olawumi, S. O. (2014).Effect of short-term feed restriction on production traits of brown and black plumage commercial layer strains at late phase of egg production. American Journal of Agriculture and Forestry, 2(2): 33-38.

Osman A. M. A., Toson M. A., Abdel-Latif S. A., Hassanien H. H. M. and T. M. A. Marwan (2010). Effect of fasting on reproductive performance of laying hens". Egypt Poultry Sci., 30(4): 1031-1057.

NRC(1994). Nutrient Requirements Of Poultry. $8^{\text {th }}$ ed. National Academy Press, Washington, DC, USA.

Powell, S. R. (2000). The antioxidant properties of zinc. J. Nutr., 1452- 1456.

Rai, M. and Ingle, A. (2012). Role of nanotechnology in agriculture with special reference to management of insect pests. Appl. Microbiology and Biotechnology, 94(2): 287-293.

Rajendran, D. (2013). Application of nano minerals in animal production system. Res. J. Biotech., 8 (3): 1-3. 
Rahman, M.M., M.A. Wahed, G.J. Fchs, A.H. Bayui and J.I. Alvarez (2002).Synergetic effect of zinc and vitamin A on the biochemical indexes of vitamin A nutrition in children. Am. J. Clin. Nutr., 1: 92-98.

Renema, R. A., and Robinson, F. E. 2004.Defining normal: comparison of feed restriction and full feeding of female broiler breeders. Worlds Poult. Sci. Journal, 60:508-522.

Renema, R.A., Robinson, F. E., Proudman, J., Newcombe, A. M. and Mckay, R. I. (1999). Effects of body weight and feed allocation during sexual maturation in broiler breeder hens.Ovarian morphology and plasma hormone profiles. Poultry Sci.,78: 629-639.

Powell, S. R. (2000). The antioxidant properties of zinc. J. Nutr. 1452-1456.

Sahoo A., Swain R.K., Mishra S.K. (2014). Effect of inorganic, organic and nano zinc supplemented diets on bioavailability and immunity status of broilers. Inter. J. Adv. Res., 2: 828-837.

Salgueiro, M. J., M. Zubillaga, A. Lysionek, M. I. Sarabia, R. Caro, T. De Paoli, A. Hager, R. Weill and J. Boccio (2000). Zinc as essential micronutrient: A review. Nutr. Res., 20(6):737 -755.

SAS. (2004). User's Guide: Statistics. Version 8.0 Cary, NC, USA.: SAS Inst.

Sauter, E.A., W.J. Stadelman, V. Harns and B.A. Mclaren (1951). Methods for measuring yolk index.Poult. Sci., 30: 629-630.

Snedecor, G.W. and W.G. Cochran (1982). Statistical Methods. $8^{\text {th }}$ ed. Iowa State Univ., Press Ames, Iowa, USA

Souza, N. A.; Oliveira, J. F.; Holanda, J. S.; Chagas, M. C. M.; Filho (2005). Ave Caipira. Natal: Orientações técnicas sobre criação de ave caipira / José Flamarion de Oliveira ... [Et al.]. - Natal [RN] : EMPARN, www.emparn.rn.gov.br, 15 pp.

Tabatabaie, M. M., Aliarabi, H., Saki, A. A., Ahmadi, A. and Siyar, H. S. A. (2007). Effect of different sources and levels of zinc on egg quality and laying hen performance.Pak. J. Biol. Sci. 10(19): 3476-3478.

Yang, X., Zhong, L., An, X., Zhang, N., Zhang, L., Han, J., Yao, J., Cote, C. and Sun, Y. (2012). Effects of diets supplemented with zinc and manganese on performance and related parameters in laying hens. Anim. Sci. Journal, 83(6): 474-81. 
تاثير نظام التحديد الغذائي وصور الزتك علي بعض الصفات الانتاجية وصفات جودة البيض والكفاعة الاقتصادية لاجاج المندرة البياض. لإن.

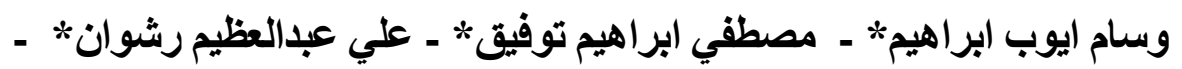

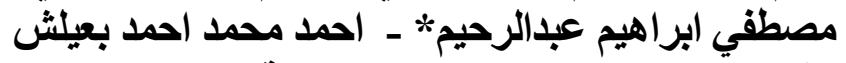

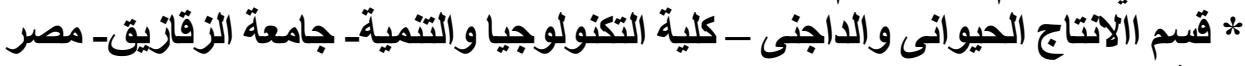
** قسم بحوث الدواجن باتثاص - معهد بحوث الاتتاج الحيوانى - الجيزة - مصر.

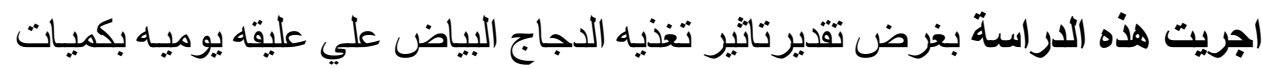

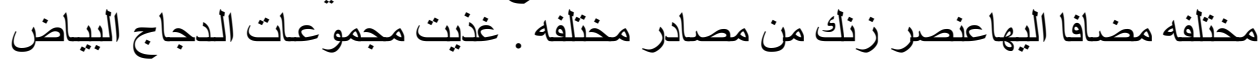

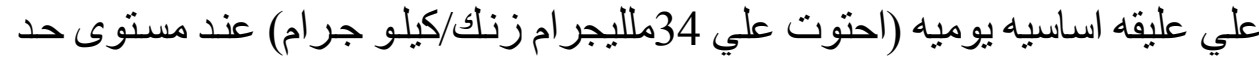

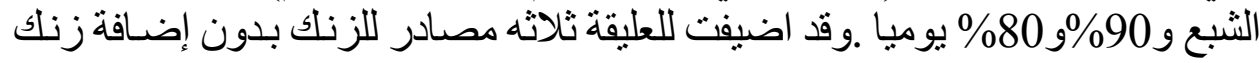

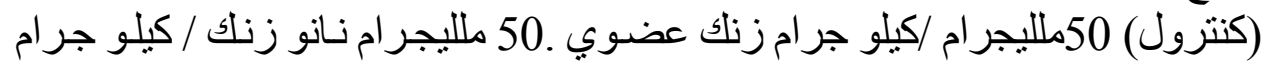

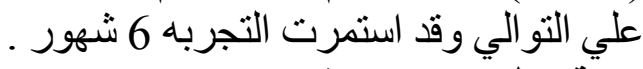
وقد اظظهرت نتائج الاراسه الاتي:

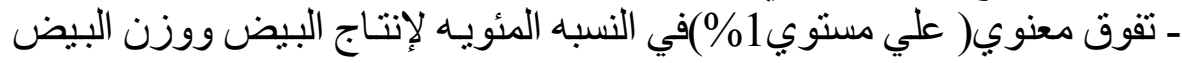

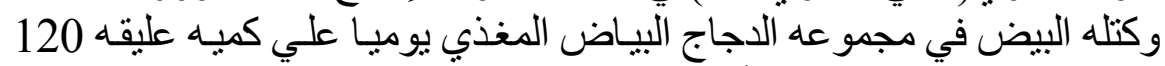

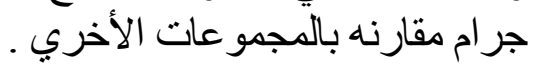

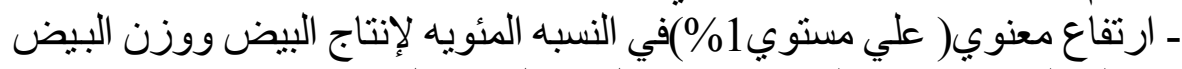

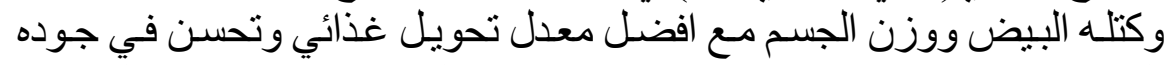

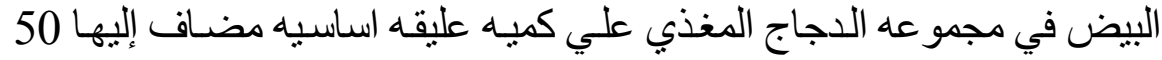

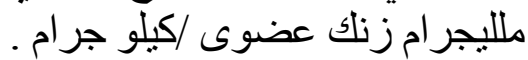

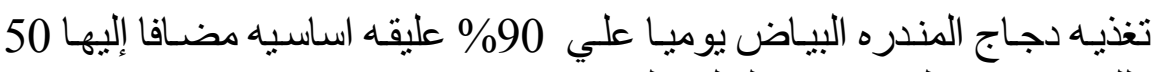

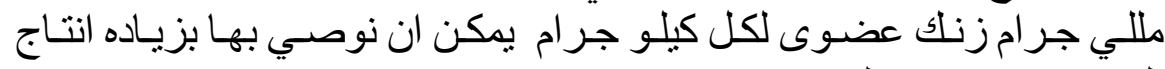
البيض وجودنه من الناحيه الاقتصاديه. 